\title{
SPACES OF SMOOTH FUNCTIONS ON ANALYTIC SETS
}

\author{
BY G. S. WELLS
}

Communicated by Samuel Eilenberg, October 25, 1976

1. Stability. Let $X \supset Y$ be real analytic (or, more generally, closed semianalytic) subsets of $R^{n}$ with $\operatorname{dim} X<n$, and let $M \subset N$ be submodules of $\left(C^{\infty}\left(R^{n}\right)\right)^{m}$ obtained (as modules of global sections) on tensoring by $C^{\infty}\left(R^{n}\right)$ coherent real analytic subsheaves $\widetilde{M} \subset \widetilde{N}$ of $\left(O\left(R^{n}\right)\right)^{m}$, where $O\left(R^{n}\right)$ denotes the sheaf of real analytic functions on $R^{n}$. Let $M(Y, X)$ (similarly for $N$ ) be the space of $m$-tuples $\phi$ of Taylor fields on $X$ flat on $Y$ such that at each point $x \in X, \phi_{x}$ is in the formal completion $M_{x}$ of $M$ at $x$. Let $r: N\left(Y, R^{n}\right) \rightarrow$ $N(Y, X) / M(Y, X)=P(Y, X)$ denote the restriction.

THEOREM 1. There is a continuous $E: P(Y, X) \rightarrow N\left(Y, R^{n}\right)$ such that $r E$ $=1$.

Theorem 1 is proved using the approach of [1, Chapter 6], where it is shown that $r: N\left(Y, R^{n}\right) \rightarrow N(Y, X)$ is onto, with modifications as in [5]; $E$ is nonlinear.

The ideal $I$ of analytic functions vanishing on a real analytic set need not be coherent, but using a suitable decomposition of $X$ by (nonclosed) semianalytic subsets, on each of which $I$ is globally generated, Theorem 1 can be applied to give, with $E(Y, X)$ denoting the space of smooth functions on $X$ flat on $Y$.

THEOREM 2. There is a continuous $E: E(Y, X) \rightarrow E\left(Y, R^{n}\right)$, a right inverse for the restriction.

J. Mather's proof ([2, in particular, p. 283 and following $]$ ), can then be applied to give

COROLlARY 1. Infinitesimal stability implies stability for smooth proper mappings of $X$ into a manifold.

2. $G$-manifolds. Let $G$ be a compact Lie group acting linearly on $R^{n}$ and let $\phi: R^{n} \rightarrow R^{m}$ be a polynomial "Hilbert" map (i.e. $\phi$ induces a mapping from the polynomials on $R^{m}$ onto the $G$ invariant polynomials on $R^{n}$ ). Let $X \subseteq R^{n}$ be a $G$ invariant analytic set and let $C_{G}^{\infty}(X)$ denote the space of $G$ invariant smooth functions on $X$. The method of Theorem 1 (see [5]) gives

THEOREM 3. There is a continuous $E: C_{G}^{\infty}(X) \rightarrow C^{\infty}\left(R^{m}\right)$ such that $\phi^{*} E=1$.

AMS (MOS) subject classifications (1970). Primary $58 \mathrm{C} 25$. 
When $X$ is a manifold this result has also been obtained by J. Mather [8] .

COROLlARY 2. G infinitesimal stability implies stability for proper smooth $G$ invariant mappings of $X$ into a $G$-manifold of finite orbit type.

When $X$ is a manifold this result has also been obtained by V. Poenaru [3]

3. Immersions. Let $X \subset R^{n}$ be a compact real analytic set and let $p \in X$. If the maximal ideal in the local ring of $C^{\infty}$ functions on $X$ at $p$ has a minimal generating set of $t$ elements, then by the Malgrange preparation theorem there is a smooth embedding $\phi$ of $B^{t}$ into $R^{n}$ with $\phi(0)=p$ and $Z=\phi\left(B^{t}\right)$ containing a neighbourhood $V$ of $p$ in $X$; also $\operatorname{Im}(D \phi(0))=T_{p}(X)$ is independent of $\phi$, where $D \phi$ denotes the derivative of $\phi . T(X)=\bigcup_{p \in X} T_{p}(X)$, the tangent space of $X$, is a subspace of $\left.T\left(R^{n}\right)\right|_{X}$, the tangent space of $R^{n}$ restricted to $X ; T_{p}(X)$ is the fibre of $T(X)$ at $p .\left.T(X)\right|_{V}$ is a subspace of $\left.T(Z)\right|_{V}$. If $K$ is a simplicial complex and $M$ is a smooth manifold, let $L_{K}(T(X), T(M))$ denote the set of continuous maps $F$ from $K$ into the space of smooth fibrewise mappings from $T(X)$ into $T(M)$ which are linear embeddings on each fibre and such that for each $k \in K$ and $p \in X$ there are neighbourhoods $U \subset V$ of $p$ and $W \subset K$ of $k$ such that $\left.F\right|_{W}$ restricted to $\left.T(X)\right|_{U}$ may be extended to a continuous map from $W$ into the smooth fibrewise linear bundle maps from $\left.T(Z)\right|_{U}$ into $T(M)$. Two such maps are homotopic if they can be joined by an element of $L_{K \times I}(T(X), T(M))$. An immersion of $X$ in $M$ is a smooth mapping $f: X \rightarrow M$ such that for each $p \in X$ there is a neighbourhood $Q$ of $p$ such that $f^{*}: C^{\infty}(M) \rightarrow C^{\infty}(Q)$ is onto. If the space of immersions (with the Whitney topology as usual) of $X$ in $M$ is denoted by $I_{m}$ and $\left[K, I_{m}\right]$ denotes the space of continuous maps from $K$ into $I_{m}$, then

THEOREM 4. The homotopy classes of $L_{K}(T(X), T(M))$ and $\left[K, I_{m}\right]$ are put in 1-1 correspondence by the derivative when $\operatorname{dim} M-\operatorname{Max}_{p \in X}\left(\operatorname{dim} T_{p}(X)\right)$ $\geqslant 1$.

Theorem 4 may be proved by a reduction to the corresponding known result for manifolds.

4. Diffeomorphisms. Let $X \subseteq R^{n}$ be a closed semianalytic set with a semianalytic stratification such that for each $x \in X$ the dimension of the stratum through $x$ and the dimension of the space of analytic tangent vectors of $X$ at $x$ coincide. Let $D(X)$ denote the space of smooth diffeomorphisms, let $I_{s}(X)$ denote the space of smooth isotopies of $X$ and let $r(F)=F(1)$, for each $F$ belonging to $I_{s}(X)$.

THEOREM 5. There is a neighbourhood $U$ of 1 in $D(X)$ and a continuous map $E: U \rightarrow I_{s}(X)$ such that $r E=1$.

Theorem 5 is proved using the techniques of [5]. 


\section{REFERENCES}

1. B. Malgrange, Ideals of differentiable functions, Oxford Univ. Press, Cambridge; Tata Inst. of Fundamental Research, Bombay, 1967. MR 35 \#3446.

2. J. N. Mather, Stability of $C^{\infty}$ mappings. II: Infinitesimal stability implies stability, Ann. of Math. (2) 89 (1969), 254-291. MR 41 \#4852.

3. V. Poénaru, Stability of equivariant smooth maps, Bull. Amer. Math. Soc. 81 (1975), 1125-1126.

4. J.-C. Tougeron, Idéaux de fonctions différentiables, Springer-Verlag, Berlin and New York, 1972.

5. G. S. Wells, Extension theorems for smooth functions on real analytic spaces and quotients by lie groups and smooth stability (preprint).

6. - Extension theorems for smooth modules (preprint).

7. - Immersions of analytic spaces (preprint).

8. J. N. Mather, Differential invariants (preprint).

DEPARTMENT OF MATHEMATICS, UNIVERSITY OF WITWATERSRAND, JOHANNESBURG, SOUTH AFRICA 